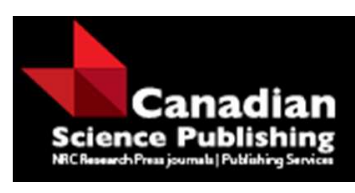

Canadian Journal of Physics

Revue canadienne de physique

\title{
Solutions of the Schrödinger equation for Pseudo-Coulomb potential plus a new Improved ring shaped potential in the cosmic string space-time
}

\begin{tabular}{|r|l|}
\hline Journal: & Canadian Journal of Physics \\
\hline Manuscript ID & cjp-2016-0066.R1 \\
\hline Manuscript Type: & Article \\
\hline Date Submitted by the Author: & 09-Mar-2016 \\
\hline Complete List of Authors: & $\begin{array}{l}\text { Ikot, Akpan; university of uyo,nigeria, } \\
\text { Abbey, Tamunoimi; Univerisity of port harcourt } \\
\text { Chukwuocha, Ephraim; uniport } \\
\text { Onyeaju, Michael; uniport }\end{array}$ \\
\hline Keyword: & $\begin{array}{l}\text { Cosmic string spacetime, Schrödinger equation, ring shaped potential, } \\
\text { Nikiforov-Uvarov method, Bound state }\end{array}$ \\
\hline \multicolumn{2}{|c}{} \\
\hline
\end{tabular}

\section{SCHOLARONE" \\ Manuscripts}




\title{
Solutions of the Schrödinger equation for Pseudo-Coulomb potential plus a new Improved ring shaped potential in the cosmic string space-time Akpan N.Ikot* ${ }^{1}$, Tamunoimi M. Abbey ${ }^{1}$, Ephraim O.Chukwuocha ${ }^{1}$ and Michael C.Onyeaju ${ }^{1}$
}

${ }^{1}$ Theoretical Physics Group, Department of Physics, University of Port Harcourt PMB 5323 Choba Port Harcourt-Nigeria

${ }^{2}$ Department of Physics, Shadrood University-Iran.

\begin{abstract}
In this paper, we obtain the bound state energy eigenvlaues and the corresponding eigenfunctions of the Schrödinger equation for the pseudo-Coulomb potential plus a new improved ring shaped like potential within the framework of cosmic string spacetime using the generalized parametric Nikiforov-Uvarov method. Our results are in good agreement with other works in the cosmic string spacetime and reduced to those in the Minkowski spacetime when $\alpha=1$.

PACS NO:03.65.Ge, 02.30.Cp,03.67.Lx

Keywords: Schrödinger equation, Nikiforov-Uvarov method, ring shaped potential , cosmic string spacetime
\end{abstract}

\section{Introduction}

The concepts of topologies play a great role in recent developments of various areas of physics such as condensed matter physics, cosmology and gauge field theory. Examples of these topological effects include quantum gravity, cosmic strings, domains wall, monopoles, branes and textures [1-3]. Cosmic strings are linear topogical defects which change the topology of the medium when viewed globally and play a significant role in cosmology. However, it has been reported that the spacetime around a cosmic string is locally and not globally[4] but the global properties of the spacetimes manifold play an important role in quantum field theories[5]. A lot of studies on the effects of topological defects on the nonrelativistc and relativistic quantum mechanical systems have been investigated such as the non-inertial effects [6], bound states of electron and holes to a disclination [7],Landau levels in the presence of topological effect [8], Coulomb and quantum oscillator problems in conical space [9], hydrogen atom in curved spacetimes [10] and others. The non-inertial effect arises from the rotating frame and gives a better understanding of the physical phenomena of the quantum theory. For instance, in quantum mechanics the non-inertial effects are linked to the coupling between the angular momentum and the angular velocity [11]. The Riemann curvature tensor is one of the tools used in the description of the topological defects in cosmic spacetimes[12] and general theory of relativity predicts that gravitation should be manifested as the curvature of spacetimes[13]. One other known example of quantum phenomena in the spacetime framework is the topological Casimic effect [14]. Casimic effect is one of the macroscopic manifestations of quantum properties of the vacuum state [15]. Recently, Bellucci et al.[16] investigated the fermionic vacuum polarization in compactified cosmic string spacetime. 
Wang et al.[17] studied the Schrödinger equation with non-central potential in the cosmic string spacetime. Afshardoost and Hassanabadi [18] studied the behaviour of a particle in the cosmic string background with noncentral potential namely Poschl-Teller double ring shaped Coulomb potential and double ring shaped oscillator. One outstanding achievement of the cosmic string in recent times is that cosmic string has ben widely used to investigate some observed phenomena such as gravitational lenses[17] Non-central potentials have been studied extensively in the literature since it provides a useful theoretical background for describing the interaction between the ring-shaped molecules and that between the deformed nucleus. The ring-shaped potentials have useful applications in quantum chemistry and are also important in nuclear physics to study rovibrational energy level of molecules, atoms and deformed nucleus [19].

In this paper, we shall consider the pseudo-Coulomb plus improved ring shaped potential,

$V(r, \theta)=-\frac{A}{r}+\frac{B}{r^{2}}+C+\frac{\hbar^{2}}{2 M r^{2}}\left(\frac{\beta \sin ^{2} \theta+\gamma \cos ^{2} \theta+\lambda}{\sin \theta \cos \theta}\right)^{2}$

where $A, B, C$ are potential parameters, and $\beta, \gamma$ and $\lambda$ are real constants. We have recently proposed the ring shaped part of Eq.(1)[20]. We plot the behaviour of the potential as a function of $r$ and $\theta$ in fig. 1

The main aim of the present paper is to attempt to investigate the behaviour of nonrelativistic particles with Pseudo-Couolmb plus new improved ring shaped potential in the cosmic string spacetime by solving the Schrödinger equation using the NikiforovUvarov(NU) method[22-23].

The paper is organized as follows. In section 2, we review the Schrödinger equation in the cosmic string spacetime. We solve the angular part of the Schrodinger equation in section 3. The solution of the radial part is given in section 4. Discussion and special cases are given in section 5. Finally, we give a brief conclusion in section 6 .

\section{Schrödinger equation in a cosmic string spacetime}

The Schrödinger equation in the cosmic string spacetime is defined as[24],

$$
i \hbar \frac{\partial \psi(r, \theta, \varphi)}{\partial t}=-\frac{\hbar^{2}}{2 M} \nabla_{L B}^{2} \psi(r, \theta, \varphi)+V(r, \theta) \psi(r, \theta, \varphi)
$$

where $\nabla_{L B}^{2}$ is the Laplace-Beltrami operator, $\mathrm{M}$ is the mass of the particle and $V(r, \theta)$ is the non-central potential. The covariant form of the Laplace-Beltrami operator is given by

$$
\nabla_{L B}^{2}=\frac{1}{\sqrt{g}} \frac{\partial}{\partial x^{\mu}}\left(\sqrt{g} g^{\mu \nu} \frac{\partial}{\partial x^{\nu}}\right)
$$

where $\mu, v=1,2,3$ and $g=\operatorname{det}\left(g_{\mu \nu}\right)$ respectively. Now for an infinitely long straight and static string, the geometry configuration produced by the spacetime is defined by the line element in spherical coordinate as,

$$
d s^{2}=-d t^{2}+d r^{2}+\alpha^{2} r^{2} \sin ^{2} \theta d \varphi^{2}
$$

where $r, \theta$ and $\varphi$ lie between the range, $0 \leq r \leq \infty, 0 \leq \theta \leq \pi, 0 \leq \varphi \leq 2 \pi$. The parameter $\alpha=1-4 G \sigma$ in Eq.(4) lie within the interval $[0,1]$ and $\sigma$ is the linear mass density of the cosmic string. As can be observed in Eq.(4) when the $\alpha \rightarrow 1$, the cosmic string spacetime 
reduced to the Minskoski spacetime. Thus the covariant cosmic string metric tensor reads,

$$
g_{\mu \nu}=\left(\begin{array}{cccc}
-1 & 0 & 0 & 0 \\
0 & 1 & 0 & 0 \\
0 & 0 & r^{2} & 0 \\
0 & 0 & 0 & \alpha^{2} r^{2} \sin ^{2} \theta
\end{array}\right), \operatorname{det}(g)=r^{4} \alpha^{2} \sin ^{2} \theta
$$

Substituting Eq.(5) into Eq.(3), we obtain the corresponding Laplace-Beltrami operator as,

$$
\begin{aligned}
\nabla_{L B}^{2} & =\frac{1}{\sqrt{r^{4} \alpha^{2} \sin ^{2} \theta}}\left\{\frac{\partial}{\partial r}\left[\alpha r^{2} \sin \theta \frac{\partial}{\partial r}\right]+\frac{\partial}{\partial \theta}\left(\frac{\alpha r^{2} \sin \theta}{r^{2}} \frac{\partial}{\partial \theta}\right)+\frac{\partial}{\partial \varphi}\left(\frac{\alpha r^{2} \sin \theta}{\alpha^{2} r^{2} \sin ^{2} \theta} \frac{\partial}{\partial \varphi}\right)\right\}, \\
& =\frac{1}{r^{2}} \frac{\partial}{\partial}\left(r^{2} \frac{\partial}{\partial}\right)+\frac{1}{r^{2} \sin \theta} \frac{\partial}{\partial}\left(\sin \theta \frac{\partial}{\partial \theta}\right)+\frac{1}{\alpha^{2} r^{2} \sin ^{2} \theta} \frac{\partial^{2}}{\partial \varphi^{2}}
\end{aligned}
$$

We now consider a non-relativistic particle influenced by the pseudo-Coulomb plus new improved ring shaped-like potential given by equation (1).Substituting Eqs.(1) and (6) into Eq.(2) yields,

$$
\begin{aligned}
& -\frac{\hbar^{2}}{2 M}\left[\frac{1}{r^{2}} \frac{\partial}{\partial}\left(r^{2} \frac{\partial}{\partial}\right)+\frac{1}{r^{2} \sin \theta} \frac{\partial}{\partial}\left(\sin \theta \frac{\partial}{\partial \theta}\right)+\frac{1}{\alpha^{2} r^{2} \sin ^{2} \theta} \frac{\partial^{2}}{\partial \varphi^{2}}\right] \psi(r, \theta, \varphi) \\
& +\left(-\frac{A}{r}+\frac{B}{r^{2}}+C+\frac{\hbar^{2}}{2 M r^{2}}\left(\frac{\beta \sin ^{2} \theta+\gamma \cos ^{2} \theta+\lambda}{\sin \theta \cos \theta}\right)^{2}\right) \psi(r, \theta, \varphi)=E \psi(r, \theta, \varphi)
\end{aligned}
$$

Taking the eigenfunction in the form,

$$
\psi(r, \theta, \varphi)=\frac{1}{\sqrt{2 \pi}} \frac{R(r)}{r} e^{i m \phi} H(\theta)
$$

and after separation of variable, we obtain the following second order differential equations,

$$
\begin{aligned}
& \frac{d^{2} R_{l l}(r)}{d r^{2}}+\left[\frac{2 M}{\hbar^{2}}\left(E+\frac{A}{r}-\frac{B}{r^{2}}-C\right)-\frac{\Lambda}{r^{2}}\right] R_{n l}(r)=0 \\
& \frac{d^{2} H_{l m}(\theta)}{d \theta^{2}}+\cot \theta \frac{d H_{l m}(\theta)}{d \theta}+\left[\Lambda-\frac{m^{2}}{\alpha^{2} \sin ^{2} \theta}-\left(\frac{\beta \sin ^{2} \theta+\gamma \cos ^{2} \theta+\lambda}{\sin \theta \cos \theta}\right)^{2}\right] H_{l m}(\theta)=0,(10)
\end{aligned}
$$

where $\Lambda$ and $m^{2}$ are the separation constants.

\section{Solution of $H(\theta)$ Angular Equation for Schrödinger Equation}

In order to solve Eq.(10), we used variable transformation, $x=\sin ^{2} \theta$ since $0 \leq \theta \leq 2 \pi$ rather than $x=\cos ^{2} \theta$ whose range lies between $0 \leq \theta \leq \pi$ and equation (10) becomes, 


$$
\begin{aligned}
& \frac{d^{2} H(x)}{d x^{2}}+\frac{1-\frac{3}{2} x}{x(1-x)} \frac{d H(x)}{d x}+ \\
& \frac{1}{x^{2}(1-x)^{2}}\left\{-\left(\frac{\Lambda}{4}+\frac{(\beta-\gamma)^{2}}{4}\right) x^{2}+\left(\frac{\Lambda}{4}+\frac{2(\gamma+\lambda)(\gamma-\beta)}{4}+\left(\frac{m}{2 \alpha}\right)^{2}\right) x-\left(\frac{(\gamma+\lambda)^{2}}{4}+\left(\frac{m}{2 \alpha}\right)^{2}\right)\right\} H(x)=0
\end{aligned}
$$

Equation is amenable to the parametric Nikforov-Uvarov method (NU)[22-23,25,26-27].

Comparing Eq.(11) with Eq.(A.1), we obtain the following,

$$
\begin{aligned}
& \alpha_{1}=1, \alpha_{2}=\frac{3}{2}, \alpha_{3}=1, \xi_{1}=\frac{\Lambda}{4}+\frac{(\beta-\gamma)^{2}}{4} \\
& \xi_{2}=\frac{\Lambda}{4}+\frac{2(\gamma+\lambda)(\gamma-\beta)}{4}+\left(\frac{m}{2 \alpha}\right)^{2}, \xi_{3}=\frac{(\gamma+\lambda)^{2}}{4}+\left(\frac{m}{2 \alpha}\right)^{2}
\end{aligned}
$$

and other coefficients are obtained from Eq.(A.4) as,

$$
\begin{aligned}
& \alpha_{4}=0, \alpha_{5}=-\frac{1}{4}, \alpha_{6}=\frac{\Lambda}{4}+\frac{(\beta-\gamma)^{2}}{4}+\frac{1}{16}, \alpha_{7}=-\frac{\Lambda}{4}-\frac{2(\gamma+\lambda)(\gamma-\beta)}{4}-\left(\frac{m}{2 \alpha}\right)^{2} \\
& \alpha_{8}=\frac{(\gamma+\lambda)^{2}}{4}+\left(\frac{m}{2 \alpha}\right)^{2}, \alpha_{9}=\frac{1}{16}+\frac{(\beta-\gamma)^{2}}{4}-\frac{(\gamma+\lambda)(\gamma-\beta)}{4}+\frac{(\gamma+\lambda)^{2}}{4} \\
& \alpha_{10}=1+\sqrt{(\gamma+\lambda)^{2}+\left(\frac{m}{\alpha}\right)}, \alpha_{11}=2+2\left(\sqrt{\frac{1}{16}+\frac{(\beta-\gamma)^{2}}{4}-\frac{(\gamma+\lambda)(\gamma-\beta)}{4}+\frac{(\gamma+\lambda)^{2}}{4}}+\sqrt{\frac{(\gamma+\lambda)^{2}}{4}+\left(\frac{m}{2 \alpha}\right)^{2}}\right) \\
& \alpha_{12}=\sqrt{\frac{(\gamma+\lambda)^{2}}{4}+\left(\frac{m}{2 \alpha}\right)^{2}}, \\
& \alpha_{13}=-\frac{1}{4}-\frac{1}{4}\left(\sqrt{1+4(\beta-\gamma)^{2}-8(\gamma+\lambda)(\gamma-\beta)+4(\gamma+\lambda)^{2}}+\sqrt{4(\gamma+\lambda)^{2}+4\left(\frac{m}{\alpha}\right)^{2}}\right)
\end{aligned}
$$

Using the eigenvalue equation of the NU method of Eq.(A.3), we obtain the relationship between the separation constant $\Lambda$ and the non-negative integer $n=n_{r}$ as,

$$
\begin{aligned}
& \Lambda=\left(2 n_{r}+1+2 \sqrt{\frac{(\gamma+\lambda)^{2}}{4}+\left(\frac{m}{2 \alpha}\right)^{2}}\right)^{2} \\
& +4\left(2 n_{r}+1+2 \sqrt{\frac{(\gamma+\lambda)^{2}}{4}+\left(\frac{m}{2 \alpha}\right)^{2}}\right) \sqrt{\frac{1}{16}+\frac{(\beta-\gamma)^{2}}{4}-\frac{(\gamma+\lambda)(\gamma-\beta)}{4}+\frac{(\gamma+\lambda)^{2}}{4}}-(\gamma+\lambda)(\beta+\lambda)
\end{aligned}
$$

Equation (14) is the contribution of the angle-dependent part of pseudo-Coulomb plus new improved ring shaped like potential. . However, when the ring-shaped term potential vanishes, that is $\gamma=\beta=\lambda=0$, then the constant of separation becomes $\Lambda=l(l+1)$, 
where $l=2 n_{r}+1+\left|\frac{m}{\alpha}\right|, m=0,1,2 \ldots$ As can be observed in Eq.(14) the separation constant depends on the parameter $\alpha$ and when the parameter $\alpha=1$, then the curved space time changes to the Minkowski space and so is the energy spectrum. The corresponding wave function is given as,

$$
\begin{aligned}
H_{l m}(\theta) & =N_{l m}\left(\sin ^{2} \theta\right)^{\frac{(\gamma+\lambda)}{2}}\left(\cos ^{2} \theta\right)^{\frac{1}{4}-\frac{1}{4}\left(\sqrt{1+4(\gamma+\lambda)^{2}+4(\beta-\gamma)^{2}-8(\gamma+\lambda)(\gamma-\beta)}\right)} \\
& \left.\times P_{n}^{\left((\gamma+\lambda), 2+\sqrt{\frac{1}{4}+(\gamma+\lambda)^{2}+(\beta-\gamma)^{2}-2(\gamma+\lambda)(\gamma-\beta)}\right.}\right)\left(1-2 \sin ^{2} \theta\right)
\end{aligned}
$$

\section{Exact Solution of Radial Part}

To solve Eq.(9), we follow the same procedure as before and obtain the energy eigenvalues as,

$$
E_{n l m}=-\frac{M A^{2}}{2 \hbar^{2}}\left(n+\frac{1}{2}+\sqrt{\frac{2 M B}{\hbar^{2}}+\Lambda}\right)^{-2}+C
$$

Substituting Eq.(14) into Eq.(16), we obtain the complete total energy eigenvalues for the pseudo-Coulomb plus new improved ring shaped like potential as,

$E_{n l m}=-\frac{M A^{2}}{2 \hbar^{2}}(n+\sigma)^{-2}+C$

Where,

$$
\sigma=\frac{1}{2}+\sqrt{\frac{2 M B}{\hbar^{2}+\left(2 n_{r}+1+2 \sqrt{\frac{(\gamma+\lambda)^{2}}{4}+\left(\frac{m}{2 \alpha}\right)^{2}}\right)^{2}+4\left(2 n_{r}+1+2 \sqrt{\frac{(\gamma+\lambda)^{2}}{4}+\left(\frac{m}{2 \alpha}\right)^{2}}\right.}}
$$

The corresponding wave function for the radial part is obtain as follows,

$$
R_{n l}(r)=N_{n l} r^{2 \sqrt{\frac{2 M B}{\hbar^{2}}+\Lambda}} e^{-\sqrt{\frac{2 M(C-E)}{\hbar^{2}}} r^{2}} L_{n}^{\left(2 \sqrt{\frac{2 M B}{\hbar^{2}}+\Lambda-1}\right)}\left(2 \sqrt{\frac{2 M(C-E)}{\hbar^{2}}} r^{2}\right)
$$

The total wave function for the system is obtain using Eqs.(8),(15) and (19) as,

$$
\begin{aligned}
\psi_{n l m}(r, \theta, \varphi) & =N_{n l m} \frac{1}{r} r^{2 \sqrt{\frac{2 M B}{\hbar^{2}}+\Lambda}} e^{-\sqrt{\frac{2 M(C-E)}{\hbar^{2}}} r^{2}} L_{n}^{\left(2 \sqrt{\frac{2 M B}{\hbar^{2}}+\Lambda-1}\right)}\left(2 \sqrt{\frac{2 M(C-E)}{\hbar^{2}}} r^{2}\right) \\
& \times\left(\sin ^{2} \theta\right)^{\frac{(\gamma+\lambda)}{2}}\left(\cos ^{2} \theta\right)^{\frac{1}{4}-\frac{1}{4}\left(\sqrt{1+4(\gamma+\lambda)^{2}+4(\beta-\gamma)^{2}-8(\gamma+\lambda)(\gamma-\beta)}\right)} \\
\times & P_{n}^{\left((\gamma+\lambda), 2-4 \sqrt{\frac{1}{4}+(\gamma+\lambda)^{2}+(\beta-\gamma)^{2}-2(\gamma+\lambda)(\gamma-\beta)}\right)}\left(1-2 \sin ^{2} \theta\right) \frac{1}{\sqrt{2 \pi}} e^{i m \phi}
\end{aligned}
$$

When $\beta=\gamma=\lambda=B=C$, then the pseudo-Coulomb ring shape potential reduces to the Coulomb potential in the cosmic string background. Substituting these parameters into Eqs.(17) and (20), we obtain the energy eigenvalues and the corresponding wave functions in the cosmic spacetime as, 


$$
\begin{aligned}
& E_{n l m}=-\frac{M A^{2}}{2 \hbar^{2}\left(n+\frac{1}{2}+\sqrt{\left(2 n_{r}+1+2 \sqrt{\left(\frac{m}{2 \alpha}\right)^{2}}\right)^{2}+\left(2 n_{r}+1+2 \sqrt{\left(\frac{m}{2 \alpha}\right)^{2}}\right)}\right)^{2}}
\end{aligned}
$$

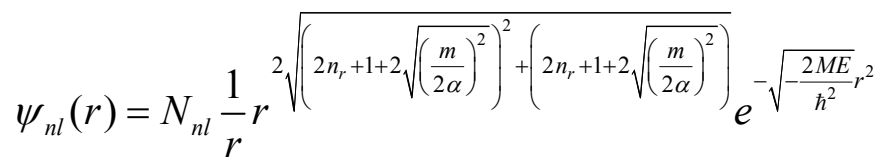

$$
\begin{aligned}
& \left.\times L_{n}^{\left(\sqrt[2]{\left(2 n_{r}+1+2 \sqrt{\left(\frac{m}{2 \alpha}\right)^{2}}\right)^{2}+\left(2 n_{r}+1+2 \sqrt{\left(\frac{m}{2 \alpha}\right)^{2}}\right.}\right)-1}\right)\left(2 \sqrt{-\frac{2 M E}{\hbar^{2}}} r^{2}\right)
\end{aligned}
$$

\section{Discussion and few Special Cases}

Let us now discuss the results and some special cases of our proposed ring shaped like potential. Now rescaling the potential parameter of our ring shaped like parameter as follows: $a \rightarrow \beta^{2}, b \rightarrow \gamma^{2}, c \rightarrow \lambda^{2}, p \rightarrow 2 \beta \gamma, 2 \beta \lambda \rightarrow q, 2 \gamma \lambda \rightarrow f$, then the PseudoCoulomb plus ring shaped like potential becomes,

$V(r, \theta)=-\frac{A}{r}+\frac{B}{r^{2}}+C+\frac{\hbar^{2}}{2 M r^{2}}\left(\frac{\left(a \sin ^{2} \theta+q+c\right)}{\cos ^{2} \theta}+\frac{\left(b \cos ^{2} \theta+f+c\right)}{\sin ^{2} \theta}+p\right)$

\subsection{Coulomb Double Ring shaped potential}

Choosing $B=C=c=b=a=p=0$ and setting $q \rightarrow-q$ and $f \rightarrow-f$, the potential (23) turns to the Coulomb ring shaped potential reported by Wang et al.[17],

$$
V(r, \theta)=-\frac{A}{r}-\frac{\hbar^{2}}{2 M r^{2}}\left(\frac{q}{\cos ^{2} \theta}+\frac{f}{\sin ^{2} \theta}\right)
$$

Now making the corresponding parameter replacements in Eqs.(17) and (20), we obtain the energy spectra and the corresponding wave function for the Coulomb Ring shaped potential in the cosmic string spacetime as,

$$
\begin{aligned}
& E_{n l m}=-\frac{M A^{2}}{2 \hbar^{2}}(n+\tilde{\sigma})^{-2} \\
& \psi_{n l m}(r, \theta, \varphi)=N_{n l m} \frac{1}{r} r^{2 \sqrt{\tilde{\Lambda}}} e^{-\sqrt{-\frac{2 M E)}{\hbar^{2}}} r^{2}} L_{n}^{(2 \sqrt{\Lambda}-1)}\left(2 \sqrt{-\frac{2 M E)}{\hbar^{2}}} r^{2}\right)\left(\sin ^{2} \theta\right)^{\frac{\sqrt{-f}}{2}}\left(\cos ^{2} \theta\right)^{\frac{1}{4}-\frac{1}{4}(\sqrt{1+4 q})} \\
& \\
& \quad \times P_{n}^{\left((\sqrt{-f}), 2-4 \sqrt{\frac{1}{4}+q}\right)}\left(1-2 \sin ^{2} \theta\right) \frac{1}{\sqrt{2 \pi}} e^{i m \phi}
\end{aligned}
$$

Where 


$$
\begin{aligned}
& \tilde{\sigma}=\frac{1}{2}+\sqrt{\left(2 n_{r}+1+2 \sqrt{-\frac{f}{4}+\left(\frac{m}{2 \alpha}\right)^{2}}\right)^{2}+4\left(2 n_{r}+1+2 \sqrt{-\frac{f}{4}+\left(\frac{m}{2 \alpha}\right)^{2}}\right) \sqrt{\frac{1}{16}+\frac{f-q}{16}-\frac{f}{4}}+\frac{f+q}{2}} \\
& \tilde{\Lambda}=\left(2 n_{r}+1+2 \sqrt{-\frac{f}{4}+\left(\frac{m}{2 \alpha}\right)^{2}}\right)^{2}+4\left(2 n_{r}+1+2 \sqrt{-\frac{f}{4}+\left(\frac{m}{2 \alpha}\right)^{2}}\right) \sqrt{\frac{1}{16}+\frac{f-q}{4}-\frac{f}{4}}+\frac{(f+q)}{2}
\end{aligned}
$$

This result is consistent with that reported by Wang et al.[17].

\subsection{A New Coulomb Ring shaped potential}

Berkedemir [27] proposed a novel angle-dependent (NAD) potential of the form,

$V_{1}(r, \theta)=-\frac{\beta}{r}+\left(\frac{\gamma+\kappa \sin ^{2} \theta+\eta \sin ^{4} \theta}{\sin ^{2} \theta \cos ^{2} \theta}\right)$

and Zhang and Hung-Fu[27] introduced a new form of NAD as,

$$
V_{2}(\theta)=-\frac{\beta}{r}+\left(\frac{\gamma+\kappa \cos ^{2} \theta+\eta \cos ^{4} \theta}{\sin ^{2} \theta \cos ^{2} \theta}\right)
$$

where $\gamma, \kappa$ and $\eta$ are real constants. If $B=C=p=0$, our ring shaped potential in Eq.(24) can be rewritten as,

$V(r, \theta)=-\frac{A}{r}+\frac{a \sin ^{4} \theta+\tau \sin ^{2} \theta+b \cos ^{4} \theta+\tau^{\prime} \cos ^{2} \theta+v}{\sin ^{2} \theta \cos ^{2} \theta}$

where $\tau=f+c, \tau^{\prime}=q+c, v=f+q+2 c$. One can observed that NAD and its new counterpart is incorporated in our proposed ring shaped like potential .If we choose $b=\tau^{\prime}=0$, the improved ring shaped potential turns in to NAD as,

$V(r, \theta)=-\frac{A}{r}+\frac{a \sin ^{4} \theta+f \sin ^{2} \theta+f}{\sin ^{2} \theta \cos ^{2} \theta}$

Making the corresponding parameter substitutions in Eqs.(17) and (20), we obtain the energy spectra and the corresponding wave function for the NAD as,

$E_{n l m}=-\frac{M A^{2}}{2 \hbar^{2}}\left(n+\sigma^{N A D}\right)^{-2}$ 


$$
\begin{gathered}
\psi_{n l m}(r, \theta, \varphi)=N_{n l m} \frac{1}{r} r^{2 \sqrt{\Lambda^{N A D}}} e^{-\sqrt{-\frac{2 M E}{\hbar^{2}}} r^{2}} L_{n}^{\left(2 \sqrt{\Lambda^{N A D}}-1\right)}\left(2 \sqrt{-\frac{2 M E}{\hbar^{2}}} r^{2}\right) \\
\times\left(\sin ^{2} \theta\right)^{\frac{\sqrt{f}}{2}}\left(\cos ^{2} \theta\right)^{\frac{1}{4}+\frac{1}{4}(\sqrt{1+4 a})} \\
\times P_{n}^{\left.(\gamma+\lambda), 2+\sqrt{\frac{1}{4}+a}\right)}\left(1-2 \sin ^{2} \theta\right) \frac{1}{\sqrt{2 \pi}} e^{i m \phi}
\end{gathered}
$$

Where,

$$
\sigma^{N A D}=\frac{1}{2}+\sqrt{\left(2 n_{r}+1+2 \sqrt{\frac{f}{4}+\left(\frac{m}{2 \alpha}\right)^{2}}\right)^{2}+4\left(2 n_{r}+1+2 \sqrt{\frac{f}{4}+\left(\frac{m}{2 \alpha}\right)^{2}}\right) \sqrt{\frac{1}{16}+\frac{a}{4}}-\frac{f}{2}}
$$

This result to the best of our knowledge has not been reported before in the cosmic string spacetime. However, when $\alpha=1$, we recover the energy spectrum and the wave function for the NAD reported in Ref.[27]. Similarly, if choose $a=\tau=0$, our proposed ring shaped potential turns into the new NAD of Eq.(30). If we substitute these parameter into the energy equation and the wave function, then we obtain the result reported in ref.[28] when $\alpha=1$.

\section{Conclusions}

We have investigated the Schrödinger equation with pseudo-Coulomb potential plus new improved ring shaped potential within the cosmic string spacetime. We employed the NU technique to obtain the discrete energy spectrum of the bound state and the corresponding wavefunctions expressed in terms of the Jacobi polynomials. As noted in ref.[17], the energy levels of the quantum system with pseudo-Coulomb plus new improved ring shaped potential in the cosmic string spacetime depend explicitly on the angular deficit $\alpha$ and characterized the global structure of the metric in the cosmic string spacetime background. Therefore, the present of the angular deficit parameter defines the curvature of the spacetime [18] and thus leads to the shift of the energy levels. The topological effect may cause changes in the gravitational field which invariably causes a change in the corresponding wave functions and energy levels .Finally, our result may find many applications in different fields of physics [29-31]

\section{Appendix A}

The NU method solves many linear second order differential equations by reducing them to a generalized equation of hypergeometric type. Here, instead of the original formulation, we use the parametric version which enables us to solve a second-order differential equation of the form [22-23,25,26]

$$
\left\{\frac{d^{2}}{d s^{2}}+\frac{\alpha_{1}-\alpha_{2} s}{s\left(1-\alpha_{3} s\right)} \frac{d}{d s}+\frac{1}{\left[s\left(1-\alpha_{3} s\right)\right]^{2}}\left[-\xi_{1} s^{2}+\xi_{2} s-\xi_{3}\right]\right\} \psi=0
$$

According to the NU method, the eigenfunctions is 
$\psi(s)=s^{\alpha_{12}}\left(1-\alpha_{3} s\right)^{-\alpha_{12}-\frac{\alpha_{13}}{\alpha_{3}}} P_{n}^{\left(\alpha_{10}-1, \frac{\alpha_{11}}{\alpha_{3}}-\alpha_{10}-1\right)}\left(1-2 \alpha_{3} s\right)$

and the energy of the system satisfies

$\alpha_{2} n-(2 n+1) \alpha_{5}+(2 n+1)\left(\sqrt{\alpha_{9}}+\alpha_{3} \sqrt{\alpha_{8}}\right)+n(n-1) \alpha_{3}+\alpha_{7}+2 \alpha_{3} \alpha_{8}+2 \sqrt{\alpha_{8} \alpha_{9}}=0$

where

$$
\begin{aligned}
& \alpha_{4}=\frac{1}{2}\left(1-\alpha_{1}\right), \quad \alpha_{5}=\frac{1}{2}\left(\alpha_{2}-2 \alpha_{3}\right), \quad \alpha_{6}=\alpha_{5}^{2}+\xi_{1}, \quad \alpha_{7}=2 \alpha_{4} \alpha_{5}-\xi_{2}, \quad \alpha_{8}=\alpha_{4}^{2}+\xi_{3} \\
& \alpha_{9}=\alpha_{3} \alpha_{7}+\alpha_{3}^{2} \alpha_{8}+\alpha_{6}, \quad \alpha_{10}=\alpha_{1}+2 \alpha_{4}+2 \sqrt{\alpha_{8}} \\
& \alpha_{11}=\alpha_{2}-2 \alpha_{5}+2\left(\sqrt{\alpha_{9}}+\alpha_{3} \sqrt{\alpha_{8}}\right), \quad \alpha_{12}=\alpha_{4}+\sqrt{\alpha_{8}} \\
& \alpha_{13}=\alpha_{5}-\left(\sqrt{\alpha_{9}}+\alpha_{3} \sqrt{\alpha_{8}}\right) \\
& P_{n}^{(\alpha, \beta)}(x)=\frac{\Gamma(\alpha+n+1)}{n ! \Gamma(\alpha+\beta+n+1)} \sum_{m=0}^{n}\left(\begin{array}{l}
n \\
m
\end{array}\right) \frac{\Gamma(\alpha+\beta+n+m+1)}{\Gamma(\alpha+m+1)}\left(\frac{x-1}{2}\right)^{m}
\end{aligned}
$$

and $P_{n}^{(\alpha, \beta)}$ is Jacobi polynomial.

\section{References}

1 E M Hu, J.Astrophys.360 L7 (1990)

2 A Vilenkin,Phys.Rept.121 263 (1985)

3 H Kleinert,Gauge Field a Condensed matter (Singapore,World Scientific ,1989)

4 H.Hassanabadi and M.Hosseinpour, Eur.Phys.J.Plus 130,236 (2015)

5 V M Mostapanenko and N N Trunov, The Casimir Efects and its Applications

(Clarendon,Oxford, 1997)

6 J.J.Sakurai,Phys.Rev.D 21,2993 (1980)

7 C Furtado and F.Moraes, Phys.Lett.A 188,394 (1994)

8 C Furtado and F.Moraes, Phys.Lett.A 19590 (1994)

9 J.Lacoelho and R L P Amaral, J.Phys.A:Math.Gen. 35,5255 (2002)

10 L.Parker,Phys.Rev.Lett.44 1599 (1980)

11 B Mashhoon, Phys.Rev.Lett.61, 2639(1988)

12 T W B Kibble, J.Phys.A: Math.Gen.9,1387 (1976), L.A.Page,Phys.Rev.Lett.35 543(1975)

13 E J Post,Rev.Mod.Phys.39,475 (1967)

14 K.A.Milton, The Casimir effect:Physical Manifestation of zero point Energy ( World Scientific, Singapore,2002)

15 E Elizade, Phys.Lett.B 516,143 (2001)

16 S. Bellucci,E.R.Bezerra de Mello,A.de Padua and A.A.Saharian, Eur.Phys.C 74,2688 (2014)

17 Z.Wang,Z.W.Long,C.Y.Long and J.Teng, Phys.Scr.90,055201 (2015) 
18 A.Afshardoost and H.Hassanabadi, Can.J.Phys.93,1 (2015)

19 C.Y.Chen, Y.You, X.H.Wang and S.H.Dong,Phys.Lett.A ,377,1521 (2013)

20 A.N.Ikot,I.O.Akpan,T.M.Abbey and H.Hassanabadi (Accepted, for publication in Communication in Theoretical Physics)

21 A F Nikiforov and V B Uvarov,Special Functions of Mathematical Physics (Birkhauser:Basel) (1988)

22 C Tezcan and R .Sever, Int. J. Theor. Phys. 48337 (2009)

23 H Hassanabadi, A N Ikot and S Zarrinkamar, Acta Phys.Polo.A 126647 (2014)

24 G.de A Marques and V.B.Bezerra, Classical Quantum Grav.19 985 (2002)

25 A.N.Ikot,H. Hassanabadi,B.Y.Yazarloo,M.I.Umo and S.Zarrinkamar, Acta Phy.Polo.A 126,656 (2014)

26 Z.Wang,Z.W.Long,C.Y.Long and L.Z.Wang,Ind.J.Phys. 89 (2015) 1059

27 C Berkdemir,J.Math.Phys.46(2009)139

28 M C Zhang,G H Sun and S H Dong,Phys.Letts.A 374(2010)704

29 M.A.Seridi and Belaloui,Int.J.Mod.Phys.A 30(2015) 1550175

30 H.Hassanabadi, A.Afshardoost and S.Zarrinkamar,Ann.Phys.356 (2015)346

31 H.Hassanabadi and M.Kamali,Chin.Phys.B 22 (2013) 100304 


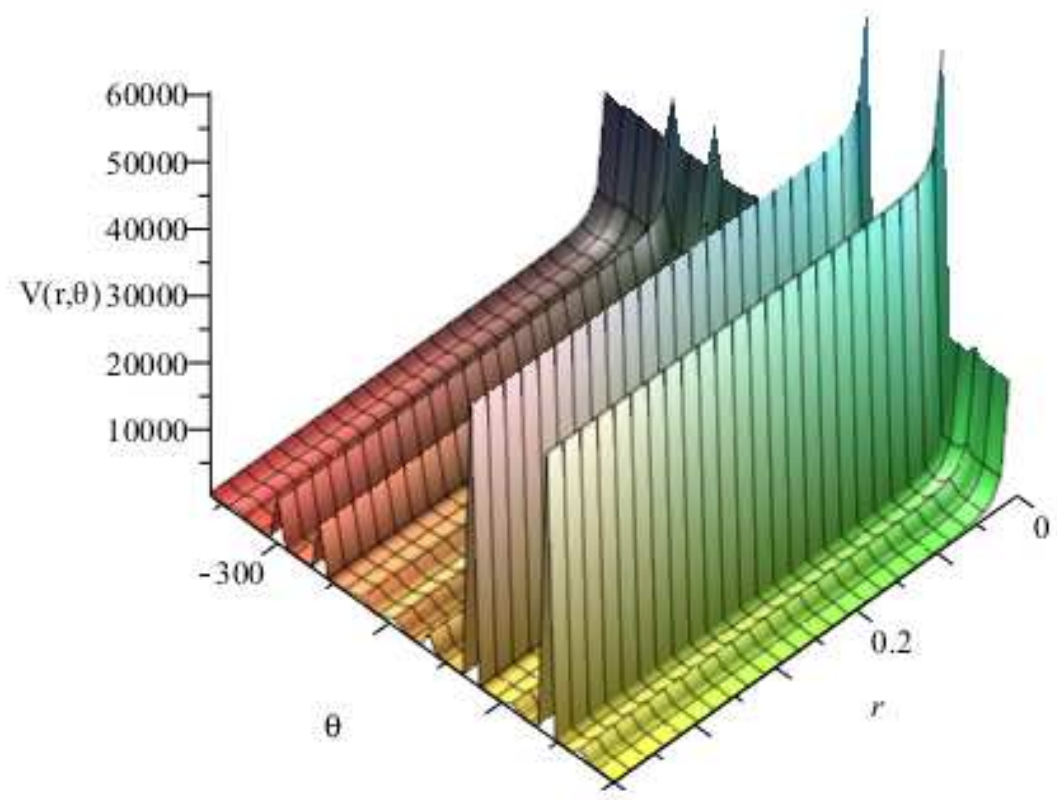

Fig.1: The plot of an expression $V(r, \theta)=-\frac{A}{r}+\frac{B}{r^{2}}+C+\frac{\hbar^{2}}{2 M r^{2}}\left(\frac{\beta \sin ^{2} \theta+\gamma \cos ^{2} \theta+\lambda}{\sin \theta \cos \theta}\right)^{2}$ as a function $r$ and $\theta$ for $\theta=(-2 \pi, 2 \pi), r=[0,5], A=-1, B=2, C=4, \beta=0.3, \gamma=5$ and $\lambda=-7$. 